Supplement of

\title{
Cloud droplet activation properties and scavenged fraction of black carbon in liquid-phase clouds at the high-alpine research station Jungfraujoch ( $3580 \mathrm{~m}$ a.s.l.)
}

5 Ghislain $\operatorname{Motos}^{1}$, Julia Schmale ${ }^{1}$, Joel C. Corbin ${ }^{1 \mathrm{a}}$, Robin Modini ${ }^{1}$, Nadine Karlen ${ }^{1 \mathrm{~b}}$, Michele Bertò $^{1}$, Urs Baltensperger ${ }^{1}$ and Martin Gysel ${ }^{1}$

${ }^{1}$ Laboratory of Atmospheric Chemistry, Paul Scherrer Institute, 5232 Villigen PSI, Switzerland

${ }^{a}$ Now at Measurement Science and Standards, National Research Council Canada, 1200 Montreal Road, Ottawa K1A 0R6, Canada

$10{ }^{b}$ Now at Institute for Aerosol and Sensor Technology, University of Applied Sciences (FHNW), Windisch, Switzerland

Correspondence to: Martin Gysel (martin.gysel@psi.ch) 
Table S1. List of all 24 cloud events sampled during the CLACE2016 field campaign and additional three cloud events of the CLACE2010 campaign included in this study. Green checkmarks are used to indicate proper instrument operation. All instruments that are not shown in this table operated correctly during the whole campaign. "min/max" refers to the minimum and maximum values of the corresponding calculated parameter during a cloud event. "Stable period ID" indicates the appellation used to refer to different stable cloud periods selected for detailed analyses. Two distinct stable periods were selected for some cloud events.

\begin{tabular}{|c|c|c|c|c|c|c|c|c|c|}
\hline $\begin{array}{c}\text { Month } \\
\text { Year }\end{array}$ & Day & $\begin{array}{c}\text { Duration } \\
\text { full } \\
\text { event } \\
{[\mathrm{h}]} \\
\end{array}$ & $\mathrm{CCNC}$ & $\begin{array}{l}\text { Inter. } \\
\text { SP2 }\end{array}$ & $\begin{array}{l}\text { PCVI } \\
\text { inlet }\end{array}$ & $\begin{array}{c}\mathrm{SS}_{\text {peak }} \\
{[\%]} \\
(\min / \max )\end{array}$ & $\begin{array}{c}T_{\text {cloud base }} \\
{\left[{ }^{\circ} \mathrm{C}\right]} \\
(\min / \max )\end{array}$ & $\begin{array}{c}\text { Stable } \\
\text { period } \\
\text { ID }\end{array}$ & $\begin{array}{l}\text { Main } \\
\text { wind } \\
\text { dir. }\end{array}$ \\
\hline $\begin{array}{l}\text { June } \\
2010\end{array}$ & 16 & 11 & $\checkmark$ & $\checkmark$ & & $0.22 / 0.40$ & $\begin{array}{c}\text { Not } \\
\text { calculated }\end{array}$ & 16June10 & $\mathrm{SE}$ \\
\hline \multirow{2}{*}{$\begin{array}{l}\text { July } \\
2010\end{array}$} & 22 & 22.5 & $\checkmark$ & $\checkmark$ & & $0.12 / 0.21$ & $\begin{array}{c}\text { Not } \\
\text { calculated }\end{array}$ & 22July10 & $\mathrm{SE}$ \\
\hline & 28 & 10.5 & $\checkmark$ & $\checkmark$ & & $0.34 / 1.37$ & $\begin{array}{c}\text { Not } \\
\text { calculated }\end{array}$ & 28July10 & NW \\
\hline \multirow{8}{*}{$\begin{array}{l}\text { June } \\
2016\end{array}$} & 16 & 18 & $\checkmark$ & & & $0.32 / 1.35$ & $-4.1 / .1 .7$ & & $\mathrm{SE}$ \\
\hline & $\begin{array}{c}18- \\
19\end{array}$ & 11 & $\checkmark$ & $\checkmark$ & & $0.21 / 0.56$ & $-6.9 /-5.2$ & $\begin{array}{c}18-19 \\
\text { June }\end{array}$ & NW \\
\hline & 21 & 22 & & $\checkmark$ & & / & $-1.3 / 2.7$ & & NW \\
\hline & 25 & 6 & $\checkmark$ & $\checkmark$ & & $0.12 / 0.24$ & $0.7 / 2.4$ & 25June & $\mathrm{SE}$ \\
\hline & 26 & 2 & $\checkmark$ & $\checkmark$ & & $0.40 / 0.44$ & $0.8 / 3.4$ & & $\mathrm{SE}$ \\
\hline & $\begin{array}{l}26- \\
27\end{array}$ & 9.5 & $\checkmark$ & $\checkmark$ & & $0.26 / 0.46$ & $-7.0 /-1.6$ & $\begin{array}{c}26-27 a \\
\& 2627 b \\
\text { June } \\
\end{array}$ & NW \\
\hline & $30 \mathrm{a}$ & 1 & & $\checkmark$ & & 1 & $0.0 / 0.4$ & & $\mathrm{SE}$ \\
\hline & $30 \mathrm{~b}$ & 1 & & $\checkmark$ & & / & $0.0 / 0.9$ & & NW \\
\hline \multirow{13}{*}{$\begin{array}{l}\text { July } \\
2016\end{array}$} & 2 & 3 & & $\checkmark$ & & 1 & $-0.6 / 0.8$ & & NW \\
\hline & 5 & 8 & $\checkmark$ & $\checkmark$ & & $0.49 / 0.76$ & $-2.0 / 1.3$ & 5July & NW \\
\hline & $8-9$ & 7 & $\sqrt{ }$ & $\checkmark$ & & $0.42 / 0.71$ & $-2.9 / 1.5$ & 8-9July & NW \\
\hline & 22 & 4 & $\checkmark$ & $\checkmark$ & $\checkmark$ & $0.29 / 0.44$ & $0.2 / 3.5$ & & $\mathrm{SE}$ \\
\hline & $23 a$ & 2 & $\checkmark$ & & & $0.25 / 0.35$ & $0.7 / 1.7$ & & SE \\
\hline & $23 b$ & 2 & $\checkmark$ & & & $0.18 / 0.28$ & $0.2 / 1.5$ & & $\mathrm{SE}$ \\
\hline & $23 c$ & 1 & $\checkmark$ & & & $0.10 / 0.18$ & $1.6 / 5.2$ & & $\mathrm{SE}$ \\
\hline & $23 d$ & 1 & $\checkmark$ & & & $0.18 / 0.26$ & $-0.1 / 1.0$ & & $\mathrm{SE}$ \\
\hline & 25 & 3.5 & $\checkmark$ & & & $0.20 / 0.31$ & $1.8 / 2.8$ & & NW \\
\hline & $27 \mathrm{a}$ & 1 & $\checkmark$ & & & $0.32 / 0.38$ & $-0.6 / 0.4$ & & NW \\
\hline & $27 \mathrm{~b}$ & 3 & $\checkmark$ & & & $0.28 / 0.66$ & $-1.5 /-0.7$ & & NW \\
\hline & $27 \mathrm{c}$ & 1.5 & $\checkmark$ & & & $0.24 / 1.10$ & $-1.7 /-1.0$ & & NW \\
\hline & $\begin{array}{c}31- \\
1 \\
\end{array}$ & 19 & $\checkmark$ & & & $0.28 / 0.80$ & $-2.6 / 1.0$ & & NW \\
\hline \multirow{3}{*}{$\begin{array}{c}\text { August } \\
2016\end{array}$} & 2 & 6 & $\checkmark$ & $\checkmark$ & & $0.64 / 1.11$ & $0.5 / 2.2$ & $\begin{array}{c}2 \mathrm{a} \& 2 \mathrm{~b} \\
\text { August }\end{array}$ & NW \\
\hline & 4 & 7 & $\checkmark$ & $\checkmark$ & $\checkmark$ & $0.19 / 0.45$ & $-0.1 / 4.1$ & 4August & $\mathrm{SE}$ \\
\hline & $5-6$ & 27 & $\checkmark$ & $\checkmark$ & & $0.54 / 0.73$ & $-5.0 /-1.1$ & $\begin{array}{c}5-6 \mathrm{a} \& \\
5-6 \mathrm{~b} \\
\text { August } \\
\end{array}$ & NW \\
\hline
\end{tabular}




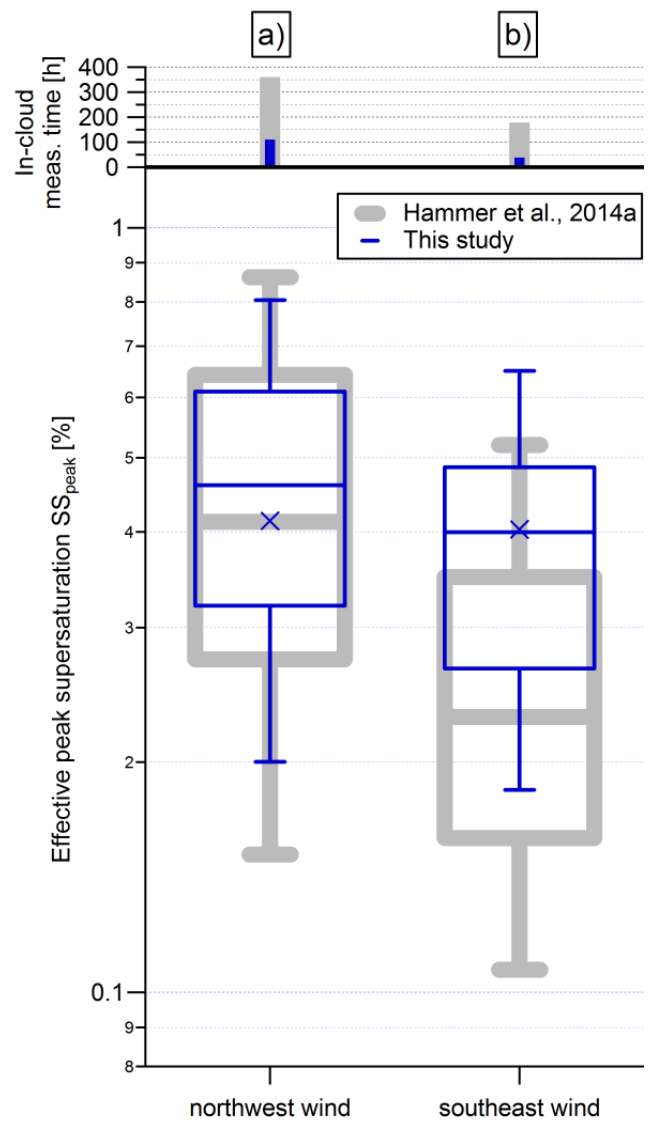

Figure S1. Boxplots representing the $10^{\text {th }}, 25^{\text {th }}, 50^{\text {th }}$ (median), $75^{\text {th }}, 90^{\text {th }}$ percentiles and the geometric mean (cross) of $\mathrm{SS}_{\text {peak }}$ of all liquid cloud events sampled during the campaign together with the cumulated time of in-cloud measurements used to produce the boxplots. The data are split between northwestern (panel (a), 478 data points) and southeastern (panel (b), 129 points) wind conditions. Grey shadings indicate the statistics of 5 previous CLACE campaigns $(2000,2002,2004,2010,2011)$ for northwest wind and 4 campaigns (same except for CLACE2000) for southeast wind. 

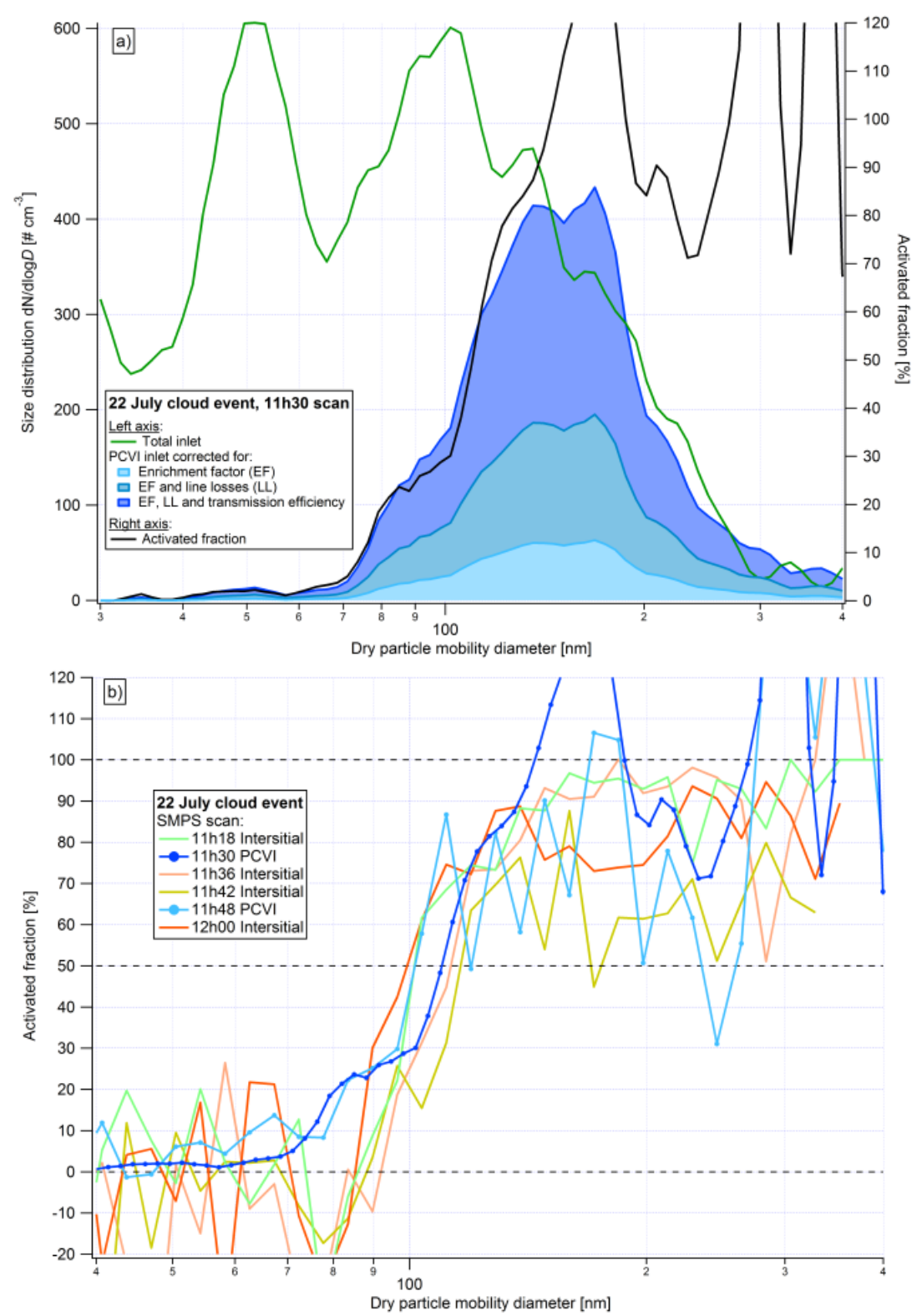

Figure S2. Cloud droplet residual particle measurements using the PCVI inlet for the example of the 22 July cloud event a) Particle number size distributions measured behind the total and PCVI inlets and corresponding activated fraction (Eq. 2). The corrections applied to the PCVI data are illustrated with the blue shadings. b) Comparison between PCVI-derived and interstitial-derived activated particle fractions. 
Coefficients of the manually fitted Hill equations (Fig. 8):

$$
f(x)=\text { base }+(\max -\text { base }) /\left(1+\left[\frac{\mathrm{x}_{\text {half }}}{\mathrm{x}}\right]^{\text {rate }}\right)
$$

For BC:

base: 0

5

rate: 1.95

xhalf: 1.55

$\max : 100$

10 For the total aerosol:

base: 0

rate: 1.8

xhalf: 0.167

max: 100 


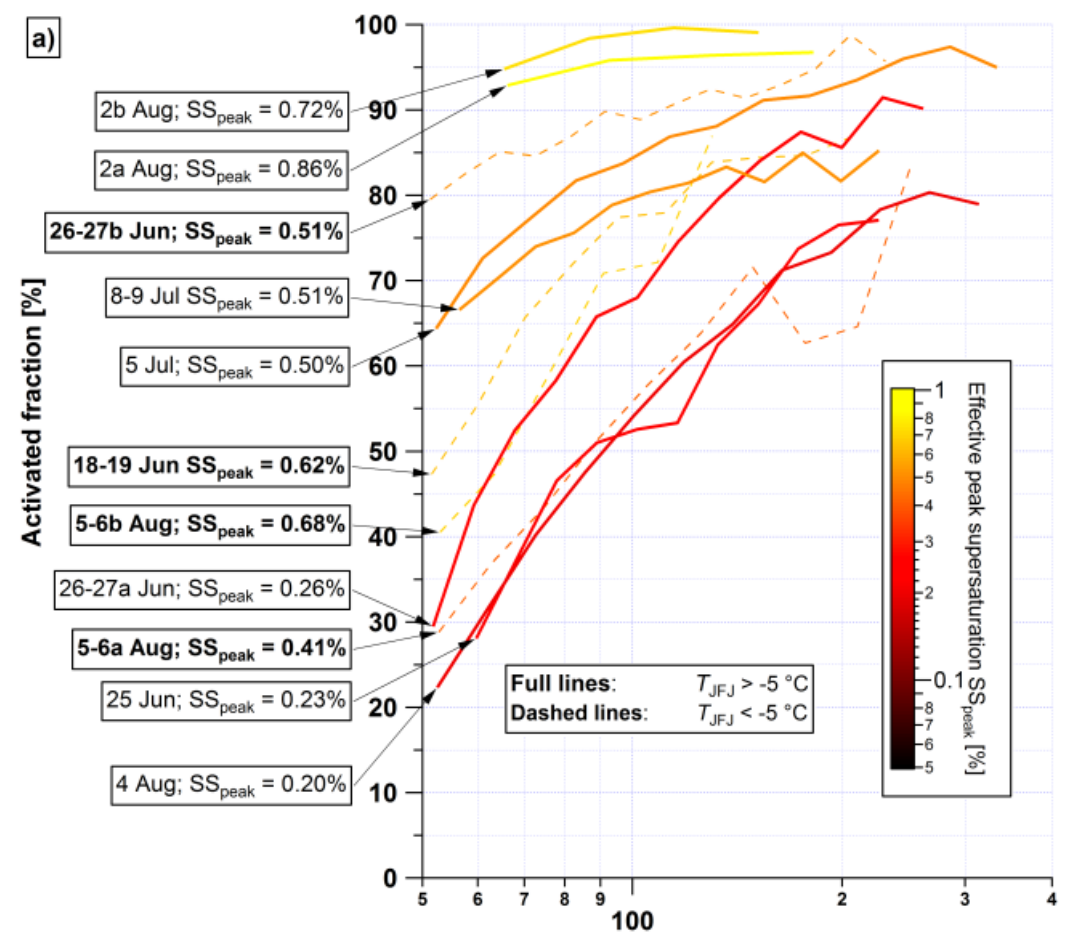

rBC mass equivalent diameter of $\mathrm{BC}$ cores [nm]

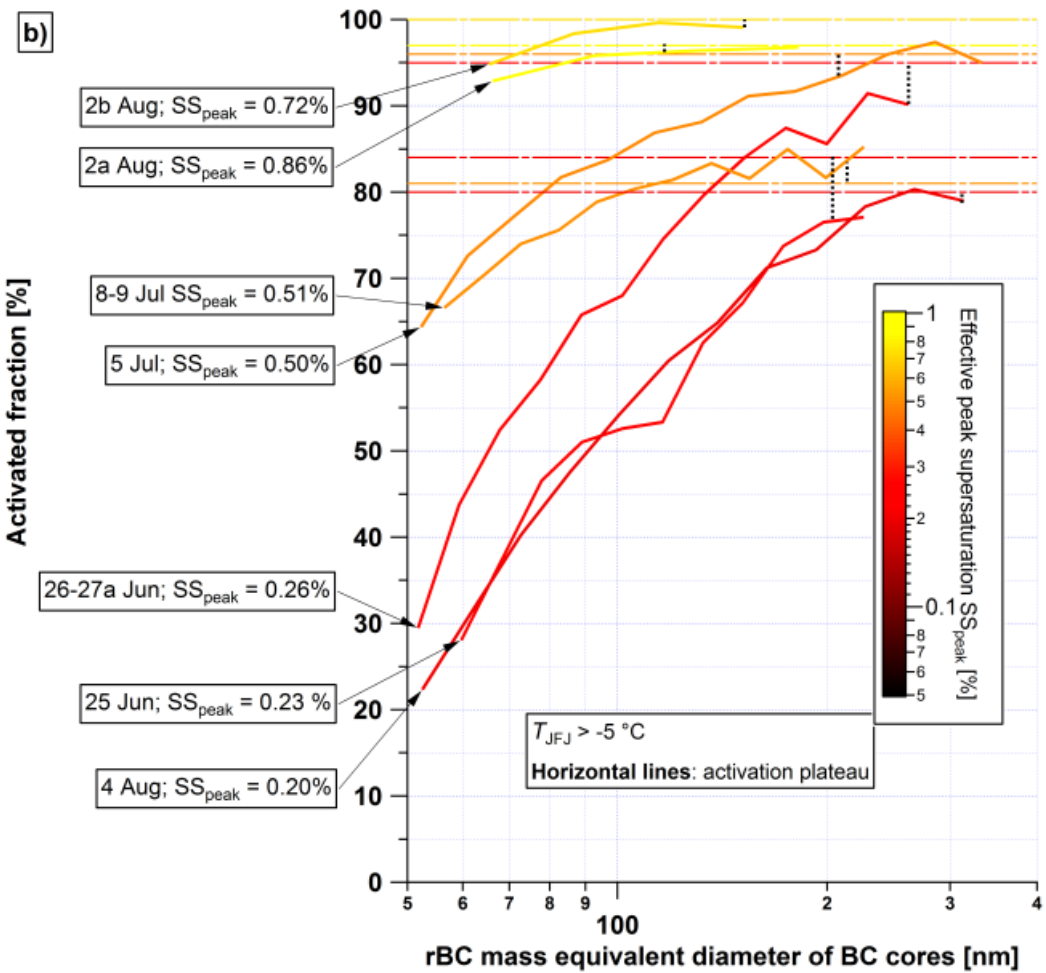

Figure S3. Variants to Figure 9, including CLACE 2016 cloud events only, averaged over the complete stable period. (a) Four stable cloud periods with $T_{\mathrm{JFJ}}$ below $-5^{\circ} \mathrm{C}$, which are likely to be mixed phase clouds and therefore expected to have lowered activated fraction across all diameters due to cloud droplet evaporation (Cozic et al., 2007; Verheggen et al., 2007), are shown as dashed lines in addition to the original figure. (b) Horizontal colored lines represent the activation plateau of all particles deduced from the SMPS data for the corresponding cloud periods with temperature above $-5^{\circ} \mathrm{C}$ (see Figure 7). 